\title{
Algorithm for Solution of Non-convex Optimization Problem through Piece-wise Convex Transformation
}

\author{
Lee Chang Kerk and Rohanin Ahmad \\ Department of Mathematical Sciences, Universiti Teknologi Malaysia \\ 81310 UTM Johor Bahru, Malaysia \\ Corresponding author: lckerk2@live.utm.my
}

Article history

Received: 29 August 2018

Received in revised form: 24 October 2018

Accepted: 7 November 2018

Published on line: 1 December 2018

\begin{abstract}
Optimization is central to any problem involving decision making. The area of optimization has received enormous attention for over 30 years and it is still popular in research field to this day. In this paper, a global optimization method called Improved Homotopy with 2-Step Predictor-corrector Method will be introduced. The method introduced is able to identify all local solutions by converting non-convex optimization problems into piece-wise convex optimization problems. A mechanism which only considers the convex part where minimizers existed on a function is applied. This mechanism allows the method to filter out concave parts and some unrelated parts automatically. The identified convex parts are called trusted intervals. The descent property and the global convergence of the method was shown in this paper. 15 test problems have been used to show the ability of the algorithm proposed in locating global minimizer.
\end{abstract}

Keywords Unconstrained optimization; convexity; globally convergent; homotopy.

Mathematics Subject Classification 65K05, 90C26

\section{Introduction}

All organizations seek efficient optimizer to help them in decision making such that the greatest benefit can be obtained from the optimized solution. Problems such as portfolio optimization often require investors to decide how much of wealth should be invested in order to get the highest return in assets [1]. These problems can be formulated as a mathematical function with or without constraints. A function can have more than one minimizer and these points are called local solutions. A local solution can be found easily by using a local search method such as Newton Raphson method with a good initial guess.

For a minimization problem, i.e, minimization of cost or risk, we seek to find a global minimizer which has the lowest function value from all solutions such that the loss can be most minimized. For a maximization problem, i.e, maximization of profit or return, a global maximizer is pursued by an individual to obtain the highest benefit possible. 
Given a real valued function $f(x)$ defined on a set $X$ in $R^{n}$, the global minimization problem to be considered is

$$
\min f(x), x \in X
$$

and we attempt to find the function value $f^{*}$ and a point $x^{*} \in X$ such that $f^{*}=\inf f$.

Global optimization is difficult, even in a one dimensional problem [2]. This is due to its nonconvex feasible region. When the objective function is non-convex, it is difficult to determine whether a potential optimum found is global. Hence, a global optimization algorithm which is able to identify an infallible bounds on all extremizers is very valuable. This kind of algorithm can be traced back to 1970 s.

Hansen [3] introduced an algorithm which applied the concept of interval analysis in 1979. This algorithm was used to solve unconstrained univariate problems. Based on the results by Hansen, the method proposed never fail to find the global minimizer if the first and second derivatives of the function have a finite number of isolated zeros .

This method was improved by Hansen in 1980 to solve multi variable global optimization problem. Hansen's method is composed of four steps [4]. The first step applied an interval version of Newton's method to locate the stationary points. The second step eliminated the parts which have greater function values than the smallest currently known function value. Sub-boxes within the feasible region will be deleted in the third step if they have monotonic property. The fourth step removes the sub-boxes which do not have a convex property.

Mohd [5] introduced a modified method from Hansen's. Like Hansen's, Mohd's method also deleted some or whole boxes which did not contain a minimizer. In [5], monotonicity test and convexity test were applied. Besides that, the currently known lowest function value was updated such that the part which has a greater function value can be deleted.

Mohd [6] later applied interval analysis to locate the region of attraction (ROA) of a function such that the global minimizer can be found. ROA is a powerful tool to optimization problems. When an ROA is recognized, we can always locate a minimizer in it by running a local search method [7].

In this paper, Improved Homotopy with 2-Step Predictor-corrector Method (I-HSPM) will be presented. Different from the methods discussed, I-HSPM does not apply interval analysis. By adopting a different technique, I-HSPM reaches the same goal which is to bound all minimizers. The details of I-HSPM will be shown in the following section.

\section{Improved Homotopy with 2-Step Predictor-corrector Method (I- HSPM)}

I-HSPM is an improved method from Homotopy Optimization with Perturbation and Ensembles(HOPE). HOPE is structured by three main parts, which are homotopy technique, perturbation tool and a local search. Generally, the global homotopy optimization methods require a big amount of computation and only applicable to the problems with small number of local minimizers. However, the capability of HOPE was shown by [8] on multi extrema problems such as 60 modal Sine function which has 60 local minimizers.

The basic concept of HOPE is to construct a simple auxiliary function with its minimizer known. Then it will use that minimizer as the initial point to locate the next minimizer on the homotopy function. A perturbation step will be applied to perturb the minimizers found 
so far in various direction. Those perturbed points are used as the next initial points to find the following minimizers. These two steps will be repeated as it deforms the auxiliary function continuously into the objective function. All the minimizers found will be stored in an ensemble.

HOPE was proved to outperformed Simulated Annealing (SA) on simple protein structure prediction problems [9]. [8] stated that, SA method converges to a solution only when the probability is almost one, while HOPE was able to converge even when the probability is less than one.

Similar to HOPE, I-HSPM also composes of three components. We replaced the perturbation tool with Intermediate Value Theorem(IVT) to change the characteristic of algorithm from stochastic to deterministic. A stochastic or probabilistic global optimization method e.g., Simulate Annealing method, HOPE method have infinite processing cycles for which the probability of having visited a global solution is not $100 \%$ guaranteed while a deterministic global optimization method will be computed in a countable number of iteration and yield a set of solutions with all global optima included [10].

IVT was introduced by Bolzano in 1917 [11]. It is a special case called Bolzano's Theorem. IVT is a common concept used in numerical analysis to determine the existence of zeroes in a function. In I-HSPM, it helps in identifying the existence of zeroes on the derivative of the objective function. The IVT is stated as follows.

Theorem 1 If a function $f$ is continuous on a closed interval $[a, b]$ and $f(a)$ and $f(b)$ have opposite signs, then there is a $c$ in $(a, b)$ for which $f(c)=K$.

Proof For proof of the theorem refer [12].

Based on IVT, let $K=0$, if $f(a)$ and $f(b)$ has opposite signs, then there exist a number $c$ in $(a, b)$ such that $f(c)=0$. Hence, we can conclude that, if sign change occurs between and $f^{\prime}(a)$ and $f^{\prime}(b)$, then at least one extremizer will lie within the interval $[a, b]$. Such interval is called the trusted interval. A trusted interval can be illustrated by a basin and works like an ROA, it will always arrive at a minimum starting from any initial guess [6].

I-HSPM is able to locate all local minimizers under the premise that all trusted intervals can be identified. IVT determines a trusted interval if the sub-interval fulfill the condition that has a negative derivative function value is followed immediately by a positive derivative function value. The following lemma shows the existence of at least a zero on the derivative function if the sign changes occur.

Lemma 1 Let $g=f^{\prime}:[a, b] \rightarrow R$. If $g$ is continuous and $g(a)<0$ and $g(b)>0$, then there exists a $c \in(a, b)$ such that $g(c)=0$

Proof By using the concept of bisection method, let a sequence of intervals be

$$
I_{0} \supseteq I_{1} \supseteq I_{2} \supseteq \ldots
$$

such that $I_{n}=\left[a_{n}, b_{n}\right], f\left(a_{n}\right)<0<f\left(b_{n}\right)$ and $b_{n}-a_{n}=\frac{b-a}{2^{n}}$.

Then

$$
\lim _{n \rightarrow \infty} \frac{b-a}{2^{n}}=0 .
$$

Hence, we have $\lim a_{n}=\lim b_{n}=c$. As $g$ is continuous, $\lim g\left(a_{n}\right)=\lim g\left(b_{n}\right)=g(c)$, with $g\left(a_{n}\right)<0$ and $g\left(b_{n}\right)>0, \forall x$. Hence, $\lim _{n \rightarrow \infty} g\left(a_{n}\right) \leq 0$ and $\lim _{n \rightarrow \infty} g\left(b_{n}\right) \geq 0$. Thus, $0 \leq g(c) \leq 0$ with $g(c)=0$. 
If zero endpoints are produced, these sub-intervals will not be selected by IVT as trusted intervals. However, minimizers might still exist within these intervals. This exclusion might lead to failure in locating true global solution. Hence, the homotopy technique is introduced to make sure this issue will not occur since if zero endpoints are produced on the target function, an approximate interval can still be determined from its slightly shifted function.

Another attempt done by modified HOPE is called Homotopy with 2-Step Predictor-corrector Method (HSPM) in 2014. HSPM can be considered as the precursor of I-HSPM, please refer [13] for details. From the simulation of [14], we noticed the influence of a small value of $\lambda$ is not strong which causes HSPM to have a lot of unnecessary function evaluations. Hence, we opted to start with larger values approaching 1 such as $0.9,0.95$ or 0.99 in I-HSPM.

The procedure of I-HSPM is as follows:

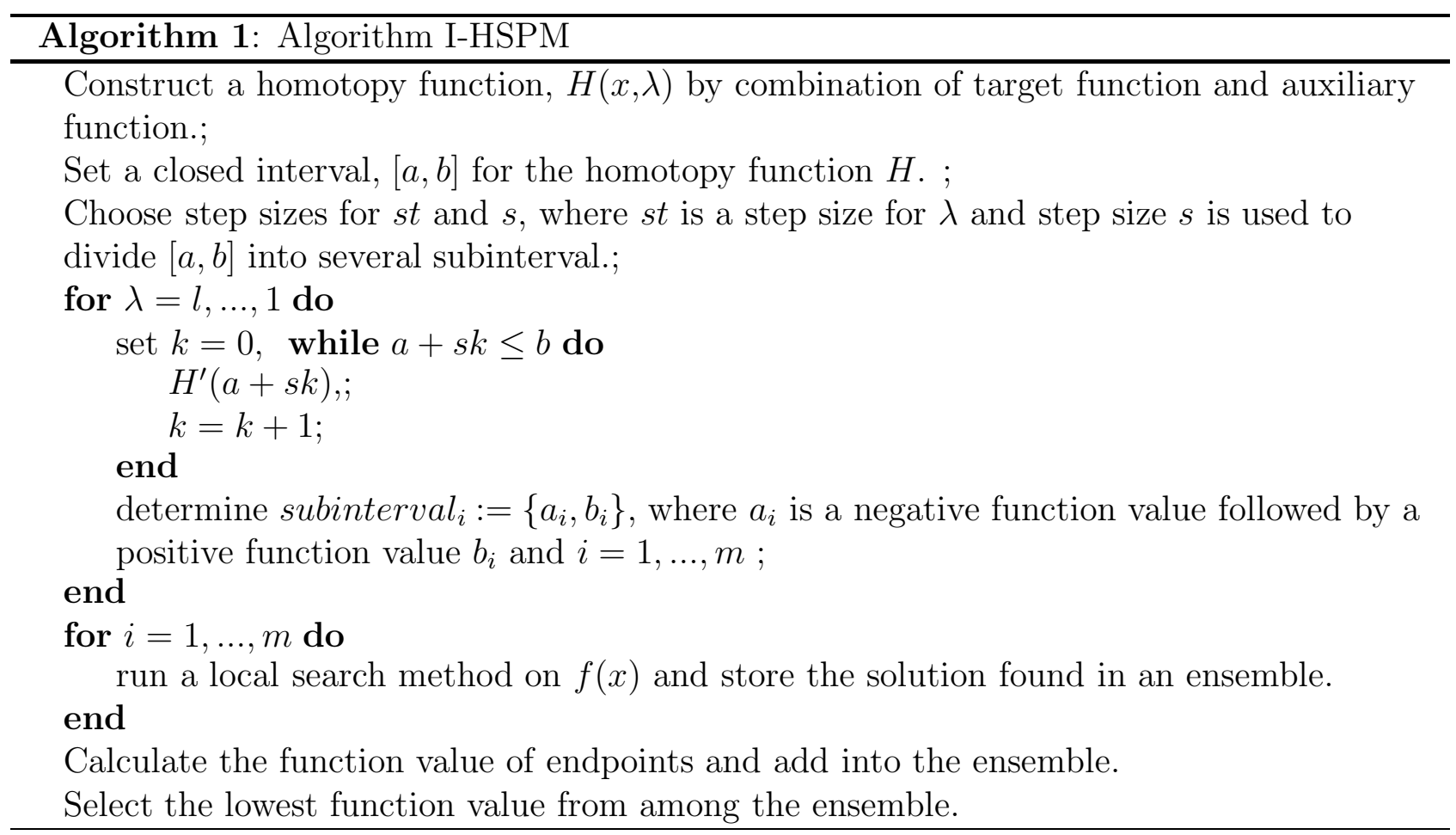

I-HSPM will first construct a homotopy function by using a simple auxiliary function and the target function. The task of the auxiliary function in I-HSPM is not like in other methods such as $[8,15-18]$, which acted like a medium to transfer solutions successively from one local to another better one. Here, the auxiliary function is used to find an approximate trusted interval when the exact trusted interval failed to be bounded.

Next, set a value for step sizes st for the homotopy function process from $l$ to 1 and $s$ to divide the closed interval $[a, b]$ into several small parts. Then, we move to the first important loop of I-HSPM, which is the identification part of the trusted interval. In this loop, all the trusted interval will be determined as $\lambda=l, \ldots, 1$.

After we gather all the trusted intervals, we enter the second important loop, which is a local search step. We will random select an initial point from each trusted interval and run a local search to find its minimizer. Each trusted interval is guaranteed to produce at least one minimizer. All the minimizers found will be stored in an ensemble. 
Finally, we compare the minimum function values from the minimizers contained in the ensemble. Hence, the global minimizer which is the lowest function value will be identified.

\section{Convexity of Trusted Interval}

In a search for sufficient condition to guarantee maxima or minima, we are lead to a class of functions called convex functions and a class of sets called convex sets [19]. Convexity is important in optimization. In general, minimizing an arbitrary function is very difficult, but if the objective function to be minimized is convex then things become considerably simpler since every local optimal solution is global if the objective function is convex [20]. Besides that, based on [21] the defining line in optimization isn't between linearity and nonlinearity, but convexity and non-convexity.

IVT technique enables I-HSPM to reduce a large non-convex optimization problem into several smaller convex problems. IVT helps I-HSPM determines all the interval which contain at least one minimizer. Trusted interval was credited in reducing the unnecessary function evaluations since the local search step will be applied only on the trusted intervals found and the same minimizer will not be located repeatedly. Since a trusted interval is expected to be convex and due to this restriction, we can say that I-HSPM is able to identify the convex parts from a non-convex feasible region.

From Section 2, a trusted interval can be determined by IVT when a negative function value is followed by a positive function value. This interval can be represented by a convex basin. The following lemma shows the convexity of the trusted interval.

Lemma 2 Let $[a, b] \subseteq R$. A function $f$ on trusted interval $[a, b]$ is strictly convex, if and only if $f^{\prime}(a)<f^{\prime}(b)$ and $f^{\prime}(a) \in(-\infty, 0)$ while $f^{\prime}(b) \in(0, \infty)$.

Proof Let $[a, b] \subseteq R$ and $a<x_{1}<x_{2}<x_{3}<x_{4}<b$.

Assume a function $f$ is monotonic decreasing on $\left(a, x_{2}\right)$ and monotonic increasing on $\left(x_{3}, b\right)$.

Then $0>f(a)>f\left(x_{1}\right)>f\left(x_{2}\right)$ and $0<f\left(x_{3}\right)<f\left(x_{4}\right)<f(b)$.

Hence $\frac{f\left(x_{2}\right)-f\left(x_{1}\right)}{x_{2}-x_{1}}<\frac{f\left(x_{4}\right)-f\left(x_{3}\right)}{x_{4}-x_{3}}$ which implies $f^{\prime}(a)<f^{\prime}(b)$.

Conversely, we will show the function is convex over a trusted interval. Let $[a, b] \subseteq R$ and $a<x_{1}<x_{2}<x_{3}<x_{4}<b$. $f^{\prime}(a)<f^{\prime}(b)$ implies

$$
\frac{f\left(x_{2}\right)-f\left(x_{1}\right)}{x_{2}-x_{1}}<\frac{f\left(x_{4}\right)-f\left(x_{3}\right)}{x_{4}-x_{3}} .
$$

Since $f^{\prime}(a) \in(-\infty, 0)$ then we can assume $0>f(a)>f\left(x_{1}\right)>f\left(x_{2}\right)$.

Similarly for the condition $f^{\prime}(b) \in(0, \infty)$, then $0<f\left(x_{3}\right)<f\left(x_{4}\right)<f(b)$.

Thus, function $f$ is monotonic decreasing on $\left(a, x_{2}\right)$ and monotonic increasing on $\left(x_{3}, b\right)$, which is convex.

Thus, Lemma 2 holds true and the trusted interval is convex. When a function is convex, it is guaranteed to have a unique global minimum [22], and it can be found by various standard methods. Hence all the local minimizers can be detected successfully by I-HSPM and thus a true global minimizer can be known. 


\section{Descent Property of I-HSPM and its Global Convergence Analysis}

Generally, an iterative algorithm which is guaranteed to generate a sequence of points converging to a limit point with any arbitrary initial point is said to be globally convergent. Here, Zangwill's global convergence theorem will be discussed. This theorem is treated as a unified manner to the study of any iterative descent algorithm [23]. Zangwill's main result is stated below.

Theorem 2 Let $A$ be an algorithm on $X$, and suppose that, given $x_{0} \in X$, the sequence $\left\{x_{n}\right\}_{k=0}^{\infty}$ is generated and satisfies $x_{k+1} \in A\left(x_{k}\right)$. Let a solution set $\Gamma \subset X$ be given, and suppose that

1. the sequence $\left\{x_{n}\right\}_{k=0}^{\infty} \supset X$ a compact set.

2. there is a continuous function $Z$ on $X$ such that

(a) if $x \notin \Gamma$ then $Z(y)<Z(x)$ for all $y \in A(x)$.

(b) if $x \in \Gamma$ then $Z(y) \leq Z(x)$ for all $y \in A(x)$.

3. the mapping $A$ is closed at all points of $X \backslash \Gamma$

Then the limit of any convergent subsequence of $\left\{x_{n}\right\}_{k=0}^{\infty}$ is a solution.

From the theorem above, two definitions follow immediately.

Definition 1 Let $X$ be a set and $x_{0} \in X$ a given point. Then an iterative algorithm $A$, with initial point $x_{0}$ is a set valued mapping $A: X \rightarrow X$ which generates a sequence $\left\{x_{n}\right\}_{k=0}^{\infty}$ according to $x_{k+1} \in A(x), n=0,1,2, \ldots$.

Definition 2 Let $\Gamma$ be the solution set of a minimization problem. Given $\Gamma \subset X$ and an iterative algorithm $A$ on $X$, a continuous real-valued function $f: X \rightarrow R$ is called descent function if

1. $x \notin \Gamma$ then $Z(y)<Z(x), \forall y \in A(x)$.

2. $x \in \Gamma$ then $Z(y) \leq Z(x), \forall y \in A(x)$.

To show I-HSPM is globally convergent by Zangwill's theorem, we need to show I-HSPM has descent property such that the second condition can be fulfilled.

A descent algorithm in optimization is an algorithm that will move to the local minimizer eventually regardless of the initial point used. Descent algorithm usually will generate a sequence $\left\{x_{n}\right\}_{n=1}^{\infty}$ which is monotonically decreasing to $\lim _{n \rightarrow \infty} f\left(x_{n}\right)$. With that sense, we have the following lemma.

Lemma 3 Let the function $f: R \rightarrow R$ closed on the trusted interval $[a, b]$ and the sequence $\left\{x_{n}\right\}_{n=1}^{\infty}$ be generated by I-HSPM. Suppose $f$ is monotonically decreasing on $\left(a, x^{*}\right)$ and monotonically increasing on $\left(x^{*}, b\right)$, where $x^{*}$ is a minimizer. Assume $a<x_{n}<x_{n+1}<\cdots<x_{m-1}<$ $x_{m}<b$. If

(a) $x_{0} \in\left(a, x^{*}\right)$, and $f\left(x_{n+1}\right)<f\left(x_{n}\right)$ or;

(b) $x_{0} \in\left(x^{*}, b\right)$, and $f\left(x_{m-1}\right)<f\left(x_{m}\right)$.

then I-HSPM is said to have the descent property. 
Proof Since the trusted interval is where the function has convex property thus it can be assumed as a basin, where the function $f$ is monotonically decreasing on the left hand side of the minimizer and monotonically increasing on the right hand side of the minimizer.

For case (a), given $x_{n}<x_{n+1}$, then

$$
\frac{f\left(x_{n+1}\right)-f\left(x_{n}\right)}{x_{n+1}-x_{n}}<0,
$$

hence $f\left(x_{n+1}\right)-f\left(x_{n}\right)<0$, thus $f\left(x_{n+1}\right)<f\left(x_{n}\right)$.

For case (b), given $x_{m-1}<x_{m}$, then

$$
\frac{f\left(x_{(m)}\right)-f\left(x_{m-1}\right)}{x_{m}-x_{m-1}}>0,
$$

hence $f\left(x_{m}\right)-f\left(x_{m-1}\right)>0$, thus $f\left(x_{m}>f\left(x_{m-1}\right)\right.$ and $f\left(x_{m-1}\right)<f\left(x_{m}\right)$. Then I-HSPM was shown has the descent property.

Based on the concept of Zangwill's convergence theory, we yield the following theorem.

Theorem 3 Let I-HSPM be an iterative algorithm on a trusted interval $X$. If the sequence generated by $I-H S P M$ has a limit point $x^{*} \in \Gamma$ for any starting point $x_{0} \in X$, where $\Gamma$ is a solution set of a minimization problem, then I-HSPM is said to be globally convergent.

Proof We will show the sequence $\left\{x_{n}\right\}_{n=1}^{\infty}$ generated by I-HSPM has a limit point $x^{*}$ such that $x_{n} \rightarrow x^{*}$ as $n \rightarrow \infty$. Suppose $f$ is a continuous descent function, then we have $f\left(x_{n}\right) \rightarrow f\left(x^{*}\right)$.

Thus, $f$ is monotonically decreasing on the sequence $\left\{x_{n}\right\}_{n=1}^{\infty}$ as follows from the property stated in Definitions 1 and 2. Hence we must have $f\left(x_{n}\right)-f\left(x^{*}\right) \geq 0$ for all $n$.

Given $\xi>0$, then $f\left(x_{n}\right)-f\left(x^{*}\right)<\xi$ which implies $x_{n} \rightarrow x^{*}$ as $n \rightarrow \infty$ and $x^{*} \in \Gamma$.

\section{$5 \quad$ Numerical Simulations}

In this section, 15 test functions (TF) are used to test the feasibility of I-HSPM and compared with others global optimization methods such as HSPM, Simulated Annealing (SA), Random Search (RS), Nelder Mead (NM) and Differential Evolution (DE). These test functions are taken from [24]. All the implementations are applied with Mathematica version 10.1 on a laptop with CPU $2.5 \mathrm{GHz}$ and $8.00 \mathrm{~GB}$ RAM.

Generally, if a function is wavy, which means it has a lot of minimizers, and it has the local minimizer that will be trapped in some smooth solver [25]. The following table shows all the test functions involved and their corresponding number of local minimizers (LoM).

Next, we compare the ability in locating the global minimizer of I-HSPM with HSPM, SA, RS, NM and DE. Except for I-HSPM and HSPM, the rest of the algorithms applied are implemented with the built-in function in Mathematica, and their corresponding control settings like number of seeds or number of perturbations are user-adjustable. Table 2 displays the result of the computation.

In Table 2, "Y" stands for "Yes, global minimizer is found", while "N" means "No, global minimizer is not found". Table 2 shows I-HSPM and HSPM are able to find the global minimizers of all 15 test functions while SA, RS, NM and DE do not. 
Table 1: Test Functions

\begin{tabular}{lllc}
\hline TF & Equation & Area of Interest & $\begin{array}{c}\text { Number } \\
\text { of LoM }\end{array}$ \\
\hline TF1 & $0.6+\sin ^{2}\left(1-\frac{16}{15}\right)-\frac{1}{50} \sin \left(4-\frac{64 x}{15}\right)-\sin \left(1-\frac{16}{15}\right)$ & $x \in[-1,1]$ & 1 \\
TF2 & $-\sin (x) \sin ^{20}\left(\frac{x^{2}}{\pi}\right)$ & $x \in[0, \pi]$ & 1 \\
TF3 & $x^{2}+\left(\frac{1}{2}\right)^{2}+\left(\frac{1}{2}\right)^{4}$ & $x \in[-5,10]$ & 1 \\
TF4 & $\left(x^{2}\right)^{\sqrt{2}}$ & $x \in[10,10]$ & 1 \\
TF5 & $-\sqrt{x} \sin (x)$ & $x \in[0.1,9.9]$ & 2 \\
TF6 & $x \sin (x)+0.1 x$ & $x \in[-6,6]$ & 3 \\
TF7 & $\sin (x)+\sin \left(\frac{10 x}{3}\right)+\log x-0.84 x+3$ & $x \in[2.7,7.5]$ & 3 \\
TF8 & $0.1\left((x-5)^{2}-\cos (5(x-5))\right.$ & $x \in[0,10]$ & 5 \\
TF9 & $-\sin (10 \log x)$ & $x \in[0.25,10]$ & 6 \\
TF10 & $1-\cos (10 x) e^{-\frac{x^{2}}{2}}$ & $x \in[-\pi, \pi]$ & 11 \\
TF11 & $10\left(x^{2}-10 \cos (2 \pi x)\right)$ & $x \in[-5.12,5.12]$ & 11 \\
TF12 & $x^{2}-10 \cos (2 \pi x)+10$ & $x \in[-5.12,5.12]$ & 11 \\
TF13 & $1-\cos (2 \pi x)+0.1 x$ & $x \in[9.9,9.9]$ & 19 \\
TF14 & $-\frac{1+\cos (12 x)}{2+0.5 x^{2}}$ & $x \in-5.12,5.12]$ & 20 \\
TF15 & $1+8 \sin \left(7(x-0.9)^{2}\right)+6 \sin ^{2}\left(14(x-0.9)^{2}\right)+(x-0.9)^{2}$ & $x \in[-5,5]$ & 230 \\
\hline
\end{tabular}

Table 2: Numerical Results

\begin{tabular}{|c|c|c|c|c|c|c|c|}
\hline $\mathrm{TF}$ & Exact Global Minimizer & I-HSPM & HSPM & $\mathrm{SA}$ & $\mathrm{RS}$ & NM & $\mathrm{DE}$ \\
\hline TF1 & -0.93279 & Y & Y & $\mathrm{Y}$ & $\mathrm{Y}$ & Y & $\mathrm{Y}$ \\
\hline TF2 & 2.20291 & Y & Y & $\mathrm{Y}$ & $\mathrm{Y}$ & Y & $\mathrm{Y}$ \\
\hline TF3 & 0 & Y & Y & $\mathrm{Y}$ & $\mathrm{Y}$ & Y & $\mathrm{Y}$ \\
\hline TF4 & 0 & Y & Y & $\mathrm{Y}$ & Y & Y & Y \\
\hline TF5 & 7.91705 & Y & Y & $\mathrm{N}$ & $\mathrm{Y}$ & $\mathrm{N}$ & $\mathrm{Y}$ \\
\hline TF6 & -4.93229 & Y & Y & $\mathrm{Y}$ & $\mathrm{Y}$ & Y & $\mathrm{Y}$ \\
\hline TF7 & 5.19978 & Y & $\mathrm{Y}$ & $\mathrm{N}$ & $\mathrm{Y}$ & Y & $\mathrm{N}$ \\
\hline TF8 & 5 & Y & Y & $\mathrm{Y}$ & Y & Y & $\mathrm{Y}$ \\
\hline TF9 & $\{0.333,0.624,1.170,2.193,4.111,7.706\}$ & Y & Y & $\mathrm{Y}$ & Y & Y & Y \\
\hline TF10 & 0 & Y & Y & $\mathrm{N}$ & $\mathrm{N}$ & $\mathrm{N}$ & $\mathrm{N}$ \\
\hline TF11 & 0 & Y & Y & $\mathrm{Y}$ & $\mathrm{Y}$ & Y & $\mathrm{Y}$ \\
\hline TF12 & 0 & Y & Y & $\mathrm{N}$ & $\mathrm{Y}$ & $\mathrm{N}$ & $\mathrm{Y}$ \\
\hline TF13 & -9.00253 & Y & Y & $\mathrm{N}$ & Y & $\mathrm{N}$ & $\mathrm{N}$ \\
\hline TF14 & -0.260024 & Y & Y & $\mathrm{N}$ & Y & Y & $\mathrm{Y}$ \\
\hline TF15 & 0.9 & Y & Y & $\mathrm{Y}$ & $\mathrm{Y}$ & $\mathrm{N}$ & $\mathrm{Y}$ \\
\hline
\end{tabular}


The Failure Rate (FR) in locating the global solution based on the result given in Table 2 by using the formula $F R=\frac{N}{T F}$ and the Success Rate (SR) by using $S R=1-F R$, are displayed in Table 3.

Table 3: Success Rate and Failure Rate

\begin{tabular}{lllllll}
\hline & I-HSPM & HSPM & SA & RS & NM & DE \\
\hline FR(\%) & 0 & 0 & 40 & 6.67 & 33.33 & 20 \\
SR $(\%)$ & 100 & 100 & 60 & 93.99 & 66.67 & 80 \\
\hline
\end{tabular}

From Table 3, RS, DE, NM and SA were not able to find the exact global solution on some test functions. Table 2 shows that, TF9 has 6 global minimizers, and all the algorithms applied are successful in locating the global solutions. However, RS, DE, NM and SA only found the one of the six solutions while HSPM and I-HSPM located all of them.

I-HSPM is an improved method from HSPM and shown has a better time complexity than HSPM [26]. Here, their CPU time will be compared by using the parameter $s=0.003$ to divide the domain into several sub-intervals. In the computation, HSPM uses $\lambda_{0}=0$ and $s t=1$, while I-HSPM applies $\lambda_{0}=1$. The result is listed in Table 4.

Table 4: CPU Time of I-HSPM and HSPM

\begin{tabular}{ccc}
\hline Test Function (TF) & I-HSPM & HSPM \\
\hline TF1 & 0.19 & 0.23 \\
TF2 & 0.39 & 0.58 \\
TF3 & 0.36 & 0.67 \\
TF4 & 0.80 & 1.17 \\
TF5 & 0.42 & 1.84 \\
TF6 & 0.39 & 0.59 \\
TF7 & 0.30 & 0.41 \\
TF8 & 0.52 & 0.67 \\
TF9 & 0.52 & 0.69 \\
TF10 & 0.91 & 1.17 \\
TF11 & 1.11 & 1.59 \\
TF12 & 1.08 & 1.61 \\
TF13 & 1.87 & 2.81 \\
TF14 & 1.69 & 2.12 \\
TF15 & 10.02 & 21.34 \\
\hline
\end{tabular}

From the results of Table 2 and 4, the reduction of CPU time will not affect the results obtained. Since the trusted interval is able to identify the region where a minimizer is located. The adjustment of value of $\lambda$ improves the CPU time without affecting the quality of global minimizer found. 
The CPU time versus TF is presented in Figure 1. It is clear that I-HSPM outperforms HSPM. The CPU time increases when the number of local minimizer increases.

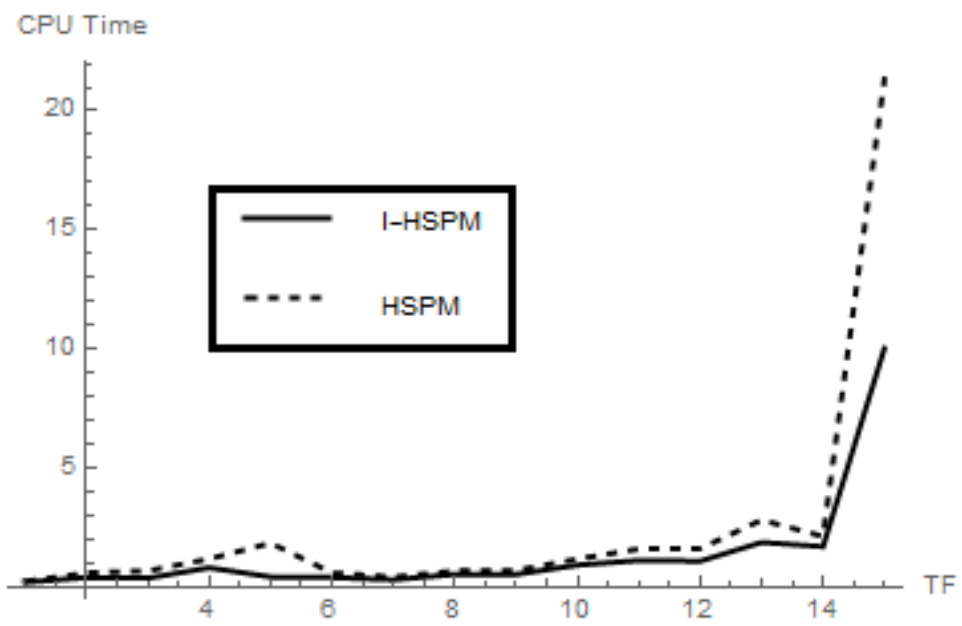

Figure 1: CPU time of I-HSPM and HSPM (dashed) versus Test Function

\section{Conclusion}

I-HSPM is an unconstrained optimization algorithm. It is able to reduce a large non-convex optimization problem into several smaller convex problems, which makes it to be unique. From the theoretical analyses, we showed that, each trusted interval found in I-HSPM is convex and I-HSPM is a globally convergent algorithm. I-HSPM ensures its capability in locating all local solutions with a small enough value of $s$, a step size which segmented the domain. When all the local minimizers are known, the global solution identified is guaranteed to be the exact global solution.

From the aspect of numerical experiments, I-HSPM and its predecessor, HSPM are able to locate all the true global solutions of all 15 test functions while some of the established algorithms failed to do so, as shown in Tables 2 and 3. Also, we can observed that, I-HSPM has lesser CPU time than HSPM particularly when the number of local minimizer increases, the CPU time needed by HSPM is higher than I-HSPM. Hence we conclude that I-HSPM is an exceptional global optimization algorithm in solving unconstrained univariate problems.

\section{Acknowledgement}

The authors would like to acknowledge the support of Universiti Teknologi Malaysia (UTM) through the GUP grant 14H58 and Mybrain 15 under Malaysia Ministry of Education.

\section{References}

[1] Kodukula, P. Organizational Project Portfolio Management: A Practitioners Guide. J. Ross Publishing. 2014. 
[2] Pintr, J. D. Global Optimization: Scientific and Engineering Case Studies. Vol. 85, Springer Science \& Business Media. 2006.

[3] Hansen, E. R. Global optimization using interval analysis: the one-dimensional case. Journal of Optimization Theory and Applications. 1979. 29(3): 331-344.

[4] Hansen, E. Global optimization using interval analysisthe multi-dimensional case. Numerische Mathematik 1980. 34(3): 247-270.

[5] Mohd, I. B. An interval global optimization algorithm for a class of functions with several variables. Journal of Computational and Applied Mathematics. 1990. 31(3): 373-382.

[6] Mohd, I. B. Identification of region of attraction for global optimization problem using interval symmetric operator. Applied mathematics and computation. 2000. 110(2): 121131.

[7] Horst, R. \& Pardalos, P. M. Handbook of Global Optimization. Vol. 2, Springer Science \& Business Media. 2013.

[8] Dunlavy, D. M. \& O'Leary, D. P. Homotopy optimization methods for global optimization.. Technical report, Sandia National Laboratories. 2005.

[9] Dunlavy, D. M., O'leary, D. P., Klimov, D. \& Thirumalai, D. HOPE: A homotopy optimization method for protein structure prediction. Journal of Computational Biology. 2005. 12(10): 1275-1288.

[10] Iwaarden, R. J. An Improved Unconstrained Global Optimization Algorithm. Ph.D.Thesis, in partial fulfillment of the requirements for the degree, Doctor of Philosophy, Applied Mathematics. University of Colorado at Denver. 1996.

[11] Grabiner, J. V. Who gave you the epsilon? Cauchy and the origins of rigorous calculus. The American Mathematical Monthly. 1983. 90(3): 185-194.

[12] Bryant, V. Mathematical analysis, by Hatton MD, Pp 242. 475 boards, 245 paperback. 1977. SBN 034020824 4/20825 2 (Hodder \& Stoughton). The Mathematical Gazette. 1977. 61(418): 307-307.

[13] Chang, K. L. \& Ahmad, R. B. Global optimization using homotopy with 2-step predictorcorrector method. in 'AIP Conference Proceedings', pp. 601-607. 2014.

[14] Kerk, L. C. Global Optimization using Homotopy with 2-Step Predictor-corrector Method. Master's Thesis. Universiti Teknologi Malaysia. 2014.

[15] Dang, C.; Talman, D. \& Wang, Z. A homotopy approach to the computation of economic equilibria on the unit simplex. Asia-Pacific Journal of Operational Research. 1999. 16(2): 155.

[16] Lin, H., Wang, Y. \& Fan, L. A filled function method with one parameter for unconstrained global optimization. Applied Mathematics and Computation. 2011. 218(7): 3776-3785.

[17] Martin, B., Goldsztejn, A., Granvilliers, L. \& Jermann, C. On Continuation Methods for Non-Linear Bi-Objective Optimization: Certified Interval-Based Approach. 2013.

[18] Renpu, G. A filled function method for finding a global minimizer of a function of several variables. Mathematical programming. 1990. 46(1-3): 191-204.

[19] Russell, D. L. Optimization Theory, Vol. 1. WA Benjamin Advanced Book Program. 1970. 
[20] Rockafellar, R. T. Some convex programs whose duals are linearly constrained. Nonlinear programming. 2014. 293-322.

[21] Rockafellar, R. T. Convex Analysis. Princeton: Princeton Univ. Press, 1970.

[22] Boyd, S. \& Vandenberghe, L. Convex Optimization. Cambridge: Cambridge University Press. 2014

[23] Du, D.-Z., Pardalos, P. M. \& Wu, W. Mathematical Theory of Optimization. Vol. 56, Springer Science \& Business Media. 2013.

[24] Gavana, A. Global optimization benchmarks and AMPGO. http://infinity r7.net/ global.optimization/test_fcn.html. 2014.

[25] Loehle, C. Global optimization using Mathematica: A test of software tools. Mathematica in Education and Research. 2006. 11(2): 139-152.

[26] Kerk, L. C. \& Ahmad, R. Improvement of time complexity of homotopy with 2-step predictor-corrector method. in 'AIP Conference Proceedings', pp. 060001. 2017. 